\title{
Metaphysics of Quantity and the Limit of Phenomenal Concepts
}

\author{
[penultimate draft: forthcoming in Inquiry]
}

\begin{abstract}
Quantities like mass and temperature are properties that come in degrees. And those degrees (e.g. $5 \mathrm{~kg}$ ) are properties that are called the magnitudes of the quantities. Some philosophers (e.g., Byrne 2003; Byrne \& Hilbert 2003; Schroer 2010) talk about magnitudes of phenomenal qualities as if some of our phenomenal qualities are quantities. The goal of this essay is to explore the anti-physicalist implication of this apparently innocent way of conceptualizing phenomenal quantities. I will first argue for a metaphysical thesis about the nature of magnitudes based on Yablo's proportionality requirement of causation. Then, I will show that, if some phenomenal qualities are indeed quantities, there can be no demonstrative concepts about some of our phenomenal feelings. That presents a significant restriction on the way physicalists can account for the epistemic gap between the phenomenal and the physical. I'll illustrate the restriction by showing how that rules out a popular physicalist response to the Knowledge Argument. [word count excluding footnotes: 7028]
\end{abstract}

Keywords: Quantity; Proportionality of Causation; Phenomenal Concept Strategy

\section{What are Magnitudes? 1}

There are two kinds of properties: those which are quantities and those which are not. Roughly speaking, quantities come in fine-grained degrees, and non-quantitative properties do not. Being prime is non-quantitative because an object is either prime or not. The property does not come in degrees. Temperature is, however, a quantity. An object does not just have temperature; it has temperature to a certain degree. The same is true for mass, temporal duration, etc. Those degrees of a quantity are magnitudes of the quantity. For example, being $5 \mathrm{~kg}$ is a magnitude.

In this essay, I assume realism about magnitudes. That is, I take the magnitudes of a quantity like all the degrees of temperature to be properties that are (i) out there in the world, (ii) not constructed by our measurement practices, and (iii) not reducible to comparative relations among objects (e.g. the relation of less-than-or-equal-to-with-respect-to-mass). ${ }^{2}$

A quantity (e.g., temperature) and its magnitudes (e.g., all the degrees of temperature) stand in a determinable-determinates relation. But not all determinates of a quantity are magnitudes of that quantity. The degrees of a quantity are maximally determinate ways to instantiate that

${ }^{\mathbf{1}}$ I received a lot of help writing this paper. I couldn't thank Ross Cameron enough for not only commenting on an earlier draft of this paper but also agreeing to supervise my dissertation that grew out of the ideas in this paper. I'm very grateful to Harold Langsam for his extensive comments and suggestions about my arguments, and for convincing me that there is something worth pursuing in the very first version of this paper. My gratitude also goes to James Cargile, Matt Duncan, Brie Gertler, Trenton Merricks, Andrew Morgan, Walter Ott, and Nick Rimell for patiently commenting on numerous versions of this paper; those conversations and feedback pushed me to think about the issue harder and articulate my reasoning more clearly. And this paper wouldn't have been half as readable as it is without Stacie Thyrion's generous help in tidying up my English. Finally, I would like to thank my audience for their valuable comments when I presented earlier drafts of this paper at Vrije Universiteit Amsterdam and Cambridge University and the anonymous reviewer of this journal for very helpful suggestions.

2 The reductive, nominalist theory of quantity is developed in Ellis (1966), Forge (1987), Bigelow \& Pargetter (1988), and recently Dasgupta (2013). For the difficulties of a nominalist theory of quantity and various ways in which a theory of quantity based on magnitude realism can be developed, see for instance Swoyer (1987), Mundy (1987), Armstrong (1988), and Eddon (2013). 
quantity. The following example supports this claim: Whereas being at 50 degrees Fahrenheit is a magnitude of temperature, being somewhere around 50 degrees Fahrenheit is a determinate but not a magnitude of temperature. By instantiating being 50 degrees Fahrenheit and being somewhere around 50 degrees Fahrenheit, a glass of water does not thereby have two magnitudes of temperature.

Furthermore, not every further specification gives us a determinate of a determinable. For example, being crimson is a determinate of redness; although being crimson and poisonous is more specific than simply being red, it is not a determinate of redness, i.e., not a more determinate way to be red. I do not have an analysis/definition of the determinable-determinate relation. In this paper, I rely largely on our intuitive understanding of it. ${ }^{3}$

\section{Some Phenomenal Qualities are Quantities}

Some philosophers speak of phenomenal qualities as quantities by talking about them coming in degrees/magnitudes (e.g., Byrne 2003; Byrne \& Hilbert 2003; Schroer 2010). It is not obvious to me that all phenomenal qualities are quantities. ${ }^{4}$ But it does seem very plausible to say that at least some sensory phenomenal qualities are. Pain, for instance, is a mental property that comes in fine-grained magnitudes like temperature and mass do. Not only can we speak of one injury being more painful than another in the way we speak of one thing being heavier than another, we can also sensibly say that, whereas being slapped in the face is just a bit more painful than being bitten by a kitten, childbirth is a whole lot more painful than being bitten by a kitten. The legitimacy of such claims supports the claim that some phenomenal qualities are quantities, whether or not we have an established procedure of measurement.

Other cases are a little bit more complicated. As Schroer points out, some phenomenal qualities are composed of multiple quantities; e.g., phenomenal colour is composed of phenomenal hue, phenomenal lightness, etc., each of which comes in degrees (2010: section 4). For the sake of simplicity, however, I'll focus on the simple cases where a phenomenal quality is a simple quantity in the subsequent discussion.

Philosophers who speak of phenomenal qualities in quantity-terms aren't very explicit about their underlying metaphysics of quantity. They don't seem to be aware of the fact that, if they were right to think that some phenomenal qualities are quantities, a proper metaphysics of quantity would have significant impact on our understanding of phenomenology. The primary goal of this essay is to explore this uncharted territory. If at least some phenomenal qualities are quanti-

\footnotetext{
${ }^{3}$ It is perhaps worth noting that the determinable-determinate relation has nothing to do with the type-token (or universal-particular) distinction. A maximally determinate property of a determinable property is still a type or universal that can be instantiated in multiple instances.

4 Even if we set the idea of cognitive phenomenology aside, whether or not all non-cognitive phenomenal qualities come in degrees, and are therefore quantities, is debatable. That is why I am making a weaker claim here. Among other things, the issue depends on what we think can be represented in our sensory experience. I am skeptical, but if, like Siegel (2010), one thinks that our visual experience can have content about, for instance, causation, and there is a causation-quale for that, such causation-quale does not seem to come in degrees, unlike the quale for pain and heat.
} 
ties, reflecting on the metaphysics of quantities generally gives us a useful cognitive anchor beyond our introspection for understanding the metaphysical nature of phenomenology.

In the following, I'll first step away from phenomenology per se to defend a metaphysical thesis about the causal profile of magnitudes generally: magnitude instantiations cannot cause concept possessions (section 3-5). Then I'll argue that, given this metaphysics of quantity, and that some phenomenal qualities are quantities, the very popular phenomenal concept strategy is not available to the physicalists for closing the explanatory gap between the mental and the physical (section 6-7).

\section{Proportionality Requirement of Causation ${ }^{5}$}

The world comes in multiple levels of determinacy. I love my omelette brown, but more specifically, golden-brown, not dark-brown. Being golden-brown is a more determinate way to be brown. Magnitudes are determinates of quantities. If we think that there are quantities and magnitudes at all, we are already committed to the metaphysical picture of reality as consisting of real layers of determinacy.

If we do not want to think that there is extensive causal over-determination, nor that the bottom level of reality monopolizes all the causal work, leaving a huge chunk of reality causally inefficacious, we need to be able to think about the levels as competing to do causal work and that the non-bottom levels can sometimes win the fight against the bottom level of determinacy. One way to get this result is by endorsing a proportionality requirement for causation (see Yablo (1997), where proportionality is used to defend the idea of wide mental causation). ${ }^{6}$

Proportionality of causation requires that for an event $E_{1}$ to be the cause of another event $E_{2}$, $\mathrm{E}_{1}$ has to be at a level of determinacy that is proportional to $\mathrm{E}_{2}$. A cause must neither be too specific nor be too general with respect to the effect. With this requirement in place, a bottom level event can lose out in a causal competition to a non-bottom level event and thereby fail to be a cause of an effect that is not at the bottom level.

The proportionality requirement can be fleshed out as the conjunction of two counterfactual conditions:

[PR1] If the instantiation of $\mathrm{F}$ is a cause of an event $\mathbf{e}$, then: for any property $\mathrm{X}$ that is a determinable of $\mathrm{F}$, it is not true that, if $\mathrm{F}$ were not instantiated but $\mathrm{X}$ were, $\mathrm{e}$ would still have happened.

[PR2] If the instantiation of $F$ is a cause of an event $e$, then: for any property $X$ that is a determinate of $\mathrm{F}$, if it were true that $\mathrm{X}$ (and hence $\mathrm{F}$ ) were instantiated, e would have happened.

\footnotetext{
5 My thoughts on a layered reality and the proportionality requirement of causation have benefited from comments and discussions with Ross Cameron and Trenton Merricks.

6 In Yablo (1992), he argues that determinables and determinates do not compete in doing causal work. They are both causes of an effect (see also Wilson (2009). In Yablo (1997), however, he thinks that there is competition. And he argues that wide mental states usually win the competition to be the cause of our actions even though the local brain states are still causally relevant.
} 
[PR1] ensures that a cause cannot be too specific. If an $\mathrm{X}$ which is more general than $\mathrm{F}$ could guarantee the presence of $\mathbf{e}$ without $\mathrm{F}$, then the instantiation of $\mathrm{F}$ is just too specific to be the cause of e. [PR2] makes sure that a cause cannot be too general. If $\mathrm{X}$ is one of the many ways in which things can be more specific than being $\mathrm{F}$ and $\mathrm{X}$ could not guarantee the presence of $\mathbf{e}$, then some way of instantiating F could not give us $\mathbf{e}$; so, the instantiation of $\mathrm{F}$ is too general to be the cause of $\mathbf{e}$.

Here is an example to illustrate the idea. Yelling 'Merry Christmas' is one among many different ways of yelling, and yelling is a way to make sound. In other words, yelling 'Merry Christmas' is a property that is a determinate of the property yelling; and the property yelling is, in turn, a determinate of the property making sound. And making sound is a determinable of yelling, and yelling is a determinable of yelling 'Merry Christmas'.

Now consider the effect that my cat is awakened. Suppose I yelled 'Merry Christmas' at it. The property yelling 'Merry Christmas' is instantiated by me. If it were not instantiated, but the more general property yelling remained instantiated (e.g. I might be yelling 'Happy Halloween' instead), my cat would still be awakened. Since the property yelling is a determinable of the property yelling 'Merry Christmas', according to [PR1], the instantiation of yelling 'Merry Christmas' is too specific to cause my cat's waking.

Since I am yelling 'Merry Christmas', obviously I instantiate the property making sound as well. Yelling 'Merry Christmas' is a determinate of the property making sound, so is whispering far away from my cat. If I were making sound but only whispering far away from my cat, my cat would not have been awakened. Thus, there is a determinate of the property making sound, such that if that determinate were instantiated and sound were still made, the effect would not have happened. So, according to [PR2], the instantiation of making sound is not specific enough to cause my cat's waking up.

In this essay, I take this proportionality requirement for causation to be a basic assumption. Some philosophers may find proportionality consideration intuitive, but are only willing to accept a proportionality constraint about the pragmatics of causal explanation, and not the metaphysics of causation. Since my motivation for admitting the proportionality requirement is metaphysical — that there shouldn't be extensive over-determination and there shouldn't be a huge chunk of reality left causally inefficacious, understanding proportionality in pure pragmatic terms wouldn't answer my metaphysical concern. That is why I take the proportionality requirement to be a metaphysical requirement for causation. An event is not a cause at all if it is not a proportional cause.

What I have just said is certainly not the final word in this discussion. One may have little sympathy for my metaphysical motivation. If so, one might prefer a pragmatic understanding of the proportionality requirement. One might even feel no intuitive pull from the idea that causation has to be proportional at all, let alone worry whether the idea should be fleshed out pragmat- 
ically or metaphysically. ${ }^{7}$ But I think that should be a debate for a separate paper. I believe I have said enough to motivate the proportionality requirement as a plausible basic metaphysical assumption. The implication of this metaphysical assumption for our understanding of phenomenal consciousness is worth exploring even if one thinks that the truth of the assumption itself can be subject to further debate.

\section{\$4 The Causal Profile of Magnitudes}

Since the goal of section 3-5 is to examine whether instantiation of any magnitude can cause our concepts, we should remain neutral at this stage for the sake of our inquiry. What I want to do is to figure out the reasonable thing to say with respect to the way magnitudes stand in causal relation with our concepts by giving a closer look at the way magnitudes stand in causal relation with other things. Setting the causes of our concepts aside, there is a good inductive reason for accepting the following principle:

[Causal Profile] For any magnitude $\mathbf{m}$, the instantiation of $\mathbf{m}$ is not a cause of the instantiation of any property $\mathrm{F}$ which is not a magnitude.

Let's consider the following case. Sound is a typical example of quantity. Suppose I am asleep. My cat jumps on my bed and meows in my ear. I am awakened. Let's name the magnitude of sound my cat made in my ear s. The question now is, given the proportionality requirement, what we should say about the causal profile of $\mathbf{s}$.

The exact same reasoning I used in the case of my yelling 'Merry Christmas' at my cat applies. Being noisy is a determinable of s. There are a lot of ways of being noisy, and instantiating that exact magnitude of sound $\mathbf{s}$ is a way to be noisy. If the magnitude of sound $\mathbf{s}$ were not instantiated and being noisy remained instantiated, I would still have been woken up. Thus, according to [PR1], the instantiation of $\mathbf{s}$ is not a cause of me waking up. For the sake of the proportionality requirement ([PR1] specifically), I cannot think of any circumstances in which instantiating $\mathbf{s}$ specifically causes my waking up.

The reasoning is not limited to the property being awakened. Cases where a specific magnitude is ruled out by [PR1] as a cause are ubiquitous. When I touched a pan freshly out of the oven, my hand was burnt. What caused the injury was the fact that the oven was extremely hot, not the very specific temperature of the oven. Of course, the pan instantiated a specific degree of temperature when I touched it and got injured. But that does not mean a specific temperature was the cause of my injury. The pan also instantiated the determinable property being extremely hot,

\footnotetext{
7 E.g. Shapiro \& Sober (2012) recently argue against both the metaphysical and pragmatic version of the proportionality constraint (what I call a metaphysical requirement here, they call it semantic). But I am not convinced by their argument. Shapiro \& Sober's argument seems to require the subjunctive conditional claim 'if not-p, then q' to mean it is impossible that not-p and q. But that is just false. The subjective conditional 'if not-p, then q' just means at the nearest possible worlds where not-p, $\mathrm{q}$ is also true at those worlds. Their counter-examples against proportionality do not seem to work once we agree to evaluate subjective conditionals by examining only the nearest possible worlds instead of all possible worlds. In any case, this is a debate for another occasion.
} 
which prevents any maximally determinate way of having temperature from being a proportional cause of my injury according to [PR1].

There are plenty of examples where a magnitude fails to qualify as a cause of an event for the same reason - [PR1]. It is instructive to observe that both being awakened and being injured are properties that are not magnitudes, i.e., not being a maximally determinate way to instantiate a property that comes in degrees. The reasoning for the causal limitation of specific magnitudes of noise and heat can be generalized and be applied not only to being awakened and being injured, but to any non-magnitude property we encounter. So, we have an inductive reason for thinking that there is always a determinable property (e.g., being approximately 100 degrees Celsius) that prevents a magnitude (e.g. being 100 degrees Celsius) from being a cause of the instantiation of a non-magnitude (e.g. boiling) due to [PR1].

One might question my inductive argument with the following example. Suppose I am tied to a machine which is designed to slap me if and only if I speak at a specific magnitude of loudness $\mathbf{~ m}$. If I speak at the volume $\mathbf{m}$ and I am slapped by the machine, it seems to be the case that it is the instantiation of $\mathbf{m}$, which is a magnitude, that proportionally causes me to be slapped. And being slapped is a non-magnitude property. So, it seems that the instantiation of magnitude can cause the instantiation of non-magnitude after all. ${ }^{8}$

First of all, I do not think we can find an actual case like that. No matter how hard we try to fine-tune our machine, I bet we won't be able to calibrate the machine so finely for it to be responsive to the instantiation of $\mathbf{m}$ specifically so that it wouldn't slap me had I spoken at any slightly different volume. More importantly, if the point of the objection is not that we can find actual cases of magnitude causing non-magnitude to be instantiated but only that such a case is conceivable, then I believe the objection is missing the point of my inductive argument. If I argue inductively that all apples are red based on my experiences with apples, one cannot challenge the inductive argument simply by imagining a black apple. [Causal Profile] is a claim about actuality. And my inductive argument is only meant to show that [Causal Profile] is true of the actual world. What else is conceivable is irrelevant.

Setting the causes of our concepts aside for the sake of our inquiry, for all the cases that we can expect to come across actually, as long as a property is not a magnitude, the instantiation of that property will not be caused by the instantiation of a magnitude, which will always be preempted by a determinable according to the requirement that causation has to be proportional. Thus, we have a good inductive reason to think that [Causal Profile] is true. As a result, we have a good reason for believing that, if having a concept is not to instantiate a magnitude, the instantiations of magnitude do not cause our possession of concepts.

\section{What Causes Our Concepts Not? ${ }^{9}$}

Having $\boldsymbol{m}$ (where $\mathbf{m}$ is a concept) is a mental property that people instantiate. Whereas having a sensory representation is to be in a sensory phenomenal state, which is arguably a magnitude,

\footnotetext{
${ }^{8}$ I would like to thank Brie Gertler for pressing me very hard on this point.

9 I couldn't emphasize enough how much this section benefits from the wonderful mix of encouraging comments and razor-sharp objections from Harold Langsam.
} 
having a concept is not a magnitude. For example, having the concept of rabbit is not a magnitude of some quantity that a person instantiates. There probably are experts about rabbits who can deploy the concept of rabbit better than others and such competence comes in degrees. But that does not mean that having the concept rabbit is a degree of instantiating some quantity.

One might protest: It depends on the content of the concept! Of course having a concept is not a magnitude if we are talking about the concept of rabbit. But if a concept $\mathbf{m}$ represents a magnitude, the property having the concept $\boldsymbol{m}$ would itself be a magnitude too. To give a concrete example, it might be argued that, since the concept $<$ being $10 \mathrm{~kg}>$ represents the magnitude being $10 \mathrm{~kg}$, the mental property having the concept $<$ being $10 \mathrm{~kg}>$ is itself a mental magnitude. ${ }^{10}$

The claim if a concept $\boldsymbol{m}$ represents a magnitude, then having the concept itself would be a magnitude is not well-motivated. Magnitudes must be magnitudes of quantities. Setting concept possession aside, for any magnitude $\mathbf{q}$ we come across in everyday life, we can tell which quantity $\mathbf{q}$ is supposed to be a magnitude of. So, it is plausible to think that, if there is a property $\mathbf{p}$ such that we cannot tell which quantity $\mathbf{p}$ is a magnitude of, $\mathbf{p}$ is not a magnitude. There is no quantity such that having a concept is a degree of that quantity. Since we cannot tell what quantity having $\boldsymbol{m}$ is a magnitude of, we have good (albeit defeasible) reason to conclude that having $\boldsymbol{m}$ is not a magnitude. When I have the concept $<$ being $10 \mathrm{~kg}>$, instantiating this mental property is not to instantiate a degree of any property that I can think of.

One may respond by suggesting that having the concept $<$ mass $>$ is a mental property which is a quantity with the property having the concept $<$ being $10 \mathrm{~kg}>$ as magnitude. So, in a similar way, as long as $\mathbf{m}$ is a concept for a magnitude, there is a quantity of which having $\boldsymbol{m}$ is a magnitude after all.

The problem with this response is that I have both the concept $<$ being $10 \mathrm{~kg}>$ and the concept $<$ being $100 \mathrm{~kg}>$ at the same time. That is, the same subject - namely I — instantiates the mental property having the concept $<$ being $10 \mathrm{~kg}>$ and the mental property having the concept $<$ being $100 \mathrm{~kg}>$ at the same time. If these two properties are meant to be magnitudes of the one quantity as the objection implies, we are in deep trouble. This is because we have one thing that instantiates more than one degree - in fact all degrees - of a quantity at the same time. And it is not as if the self has infinitely many parts which can instantiate all these mental properties separately. This is as absurd as saying that I instantiate all possible weights. Therefore, having the concept $<$ being $10 \mathrm{~kg}>$ is not a magnitude of having the concept $<$ mass $>$.

Finally, it has been suggested to me that I have overlooked the distinction between simple and complex quantities (and hence the distinction between simple and complex magnitudes). For example, temperature is a simple quantity; it just comes in various degrees. Colour, however, is a complex quantity in the sense that it is composed of multiple quantities: brightness, hue, saturation. Having a concept is presumably grounded upon a brain state, which consists of a network of electric activations in a certain area of our brain. This network of activation is composed of all the local activations. And these local electric activations are instantiations of magnitudes. So, it might be argued, just like a particular shade of colour, having a concept is a complex magnitude.

10 I use $<\mathrm{x}>$ to signify the concept of $\mathrm{x}$. And italic is reserved for properties, e.g., being massive. 
We should be careful to note that the quantities hue, saturation, and brightness form the complex quantity colour by fusing together as different dimensions of one quantity. Colour is not formed by putting an instantiation of a magnitude of hue next to an instantiation of a magnitude of brightness (whatever that even means). That, however, is exactly what's going on in the brain case: neural activations are linked next to each other to form a pattern. What we have in the case of brain activation networks is not a case of simple quantities fusing to form a complex quantity - it is a case of multiple quantity instantiations being connected next to each other. Hence, the fact that concept possessions are grounded upon networks of brain activation does not imply that having a concept is a complex magnitude like the colours.

None of the apparent objections work. Thus, I conclude that having a concept is not to instantiate a magnitude. Together with [Causal Profile], we can conclude that our concepts are beyond the causal range of magnitude instantiations. Our conceptual apparatus is simply not causally sensitive to any specific degree of a quantity. Why should we care? Here is one reason: if Byrne et al. are right to think that at least some phenomenal qualities are quantities, this metaphysics of quantity leads to a serious problem for physicalism about phenomenal consciousness.

\section{Phenomenal Qualities as Quantities Revisited}

If a phenomenal quality is to be understood as a quantity, we should conceptualize it like we conceptualize other quantities. To avoid confusion, it is better to separate the quantity from the magnitudes of the quantity in the same way we distinguish the quantity temperature and the degrees of temperature. Take pain as an example again. Pain is a quantity that comes in various degrees of painful feelings. Let us call the quantity itself a phenomenal quality and the degrees or magnitudes of the quantity phenomenal feelings. So, the phenomenal feelings are determinates of a phenomenal quality.

I have argued that magnitudes of a quantity are maximally determinate properties of the quantity. For instance, an object that is very heavy and is $100 \mathrm{~kg}$ does not thereby have two magnitudes of mass. Both being very heavy and being $100 \mathrm{~kg}$ are ways to have mass; and the object instantiates both mass-related properties. Nonetheless, the object has only one magnitude of mass, namely, the maximally determinate way to have mass, being $100 \mathrm{~kg} .{ }^{11}$

Now we take certain phenomenal feelings to be degrees or magnitudes of phenomenal qualities. So, based on what we have said about magnitudes in general, we ought to say that those phenomenal feelings are maximally determinate ways of instantiating their respective phenomenal qualities too. Accordingly, when we feel pain, although we could have felt a different degree of pain, we could not have been more determinately painful than the way we feel it. What we feel when we are in pain is always a maximally determinate way to be in pain.

If there is any lingering doubt (which is probably a philosopher's second nature), we can always run an argument that is parallel to the one that supports treating magnitudes of mass as maximally determinate ways to have mass. When I break my leg, I instantiate multiple pain-related mental properties: E.g. being very painful, being more than slightly painful, being at level 7 pain, etc. All these pain-related properties are ways to instantiate pain, only different in terms of

11 Notice that being $100.1 \mathrm{~kg}$ is not a more determinate way to have mass. It is a different maximally determinate way to have mass. 
determinacy. And I instantiate them all. Nonetheless, I feel just one pain. Suppose a doctor asks me how painful my broken leg is. It would be nonsensical for me to respond by asking, 'Which pain?' There is just one. And the metaphysics of phenomenal character should respect our phenomenal introspection. So, for all the instantiated mental properties lining up in a determinabledeterminate hierarchy of pain, only one can be identified as the mental property that is my painful feeling when I break my leg.

Here's a question: which among all the properties in the determinable-determinate hierarchy of pain should be identified as the painful feeling? The only principled and plausible choice would be the maximally determinate one. ${ }^{12}$

I cannot emphasize enough that none of what I just said is to deny that the non-maximal determinates of being in pain (e.g., being very painful) are mental properties. They are mental properties too. (You might even call them phenomenal properties if you like.) They simply aren't the subjective feelings, which are maximally determinate. A property doesn't have to be a feeling to be mental. For example, being very painful and being more than slightly painful are surely additional mental properties which I instantiate along with and in virtue of the magnitude of pain I feel when I break my leg, ${ }^{13}$ but being very painful and being more than slightly painful are not extra feelings over and above the very magnitude of pain I feel.

This is how all quantities and magnitudes work. I am simply running exactly the same kind of argument I ran for quantities like mass or temperature on the phenomenal qualities that come in fine-grained degrees. If the argument makes sense in those cases, it should make sense here. So, if a phenomenal quality comes in degrees, what we feel subjectively are magnitudes of such a quality, namely, maximally determinate ways to instantiate such a quality.

\section{Magnitudes and Phenomenal Concepts}

Philosophers refer to some of our subjective phenomenal feelings as magnitudes casually. Now that we have a better sense of how to conceptualize some of our phenomenology as quantities, we are in a position to see how the metaphysical thesis about a causal barrier between magnitudes and concepts can inform the way we understand our phenomenology. As a case study, I will focus on how the causal barrier restricts the physicalists' options in responding to Jackson's (1982) Knowledge Argument for dualism. And I assume a basic familiarity with the Knowledge Argument here.

There are various physicalist attempts to defuse the Knowledge Argument. And the phenomenal concept strategy is one of the widely accepted approaches. The basic idea of the strate-

\footnotetext{
12 One alternative suggestion that has been presented to me is, although there is only one pain that I feel, which property in the determinable-determinate hierarchy my phenomenal feeling is depends on the attention of the pain sufferer. An undesirable consequence of this suggestion is that it seems we have to say that as long as I am not paying attention, I will feel just a pain determinable while there are some more specific pains that I instantiate without feeling them. Furthermore, it also seems to me that whether a mental property is a phenomenal feeling or not should be intrinsic to that mental property and not subject to contingencies like shift of attention. I think the way I model phenomenal feelings upon magnitudes like temperatures gives us a very neat way to organize all the intuitions we have about phenomenal feelings.
}

13 Notice that this talk of 'additional mental properties' does not contradict the fact that we instantiate being very painful in virtue of instantiating a magnitude of pain. 
gy is that there are two kinds of concept we use to refer to our phenomenal feelings, and one of them (the phenomenal concepts) cannot be acquired or possessed by a person unless she has actually experienced the relevant phenomenal feeling. Since the co-extensionality of the two kinds of concept is not a priori knowable, we have the misleading intuition that Mary learns something new about the world after seeing something red for the first time. But what really happened was that Mary acquired a new concept to refer to something she has known of already.

A very popular way to employ the phenomenal concept strategy, first developed by Loar (1990), is to say that the phenomenal concepts are a kind of mental demonstrative (e.g. 'that colour experience'). To address the apparent richness of our grasp of phenomenology, advocates of the strategy sometimes beef up the demonstrative phenomenal concepts by adding extra components or extra descriptive content to them (e.g., Block 2002; Levine 2007; Schroer 2010). But the crucial element remains constant: such concepts are taken to be at least partly demonstratives. Due to the nature of demonstratives in general, the (colour) phenomenal concepts are such that Mary could not have acquired them without actually having run into colour experiences. ${ }^{14}$ That is why Mary could not possibly have the related phenomenal concepts before she first actually had real visual experience of colour.

Certainly, from time to time, we seem to be able to have mental demonstratives about things we have not encountered; but, as Levine (2010) convincingly argued, those are more likely to be cases of disguised mental definite descriptions instead of genuine demonstratives. For example, when I think about that woman who will be my future wife, I seem to be entertaining a mental demonstrative. But if in fact I have not met her yet, it is plausible to say I am in fact just thinking about the woman who will be my future wife. And the thought is not really a demonstrative. So, if phenomenal concepts are demonstratives of some sort, one needs to have had the relevant experience and have encountered the referents of the demonstratives to have the concepts at one's disposal. If the epistemic intuition that drives the Knowledge Argument is jointly produced by (i) the nature of such demonstrative phenomenal concepts and (ii) the fact that we cannot know a priori that these demonstrative concepts are co-extensive with certain neurological concepts about brain states, we do not have an argument for dualism as a metaphysical doctrine.

This is a very rough characterization of the strategy, but the key point is this: for the strategy to work, demonstrative concepts referring to phenomenal feelings must be possible. But if my metaphysical thesis about the causal barrier between magnitudes and concepts is true, the physicalists have good reason to think that we don't have such kind of concept about our phenomenal feelings. Here is why.

There are two kinds of demonstrative concepts: the simple ones and the complex ones. I can entertain the thought $<$ that is tasty $>$, which involves a simple mental demonstrative, and also the thought $<$ that bagel is tasty $>$, which involves a complex mental demonstrative. A simple demonstrative is an indispensable component of a complex demonstrative. A complex demonstrative of something is impossible if a simple demonstrative about that thing is impossible. If I can't think of something as that, I can't think of it as that bagel.

\footnotetext{
14 Phenomenal concepts understood in this way are also called recognitional concepts in the literature. For other defenders of this approach, see Tye (2003) and Caruthers (2004). Tye now renounces the phenomenal concept strategy (2009).
} 
The phenomenal concept strategy is a physicalist argument. So, we should review the situation through the physicalist lens. (Sometimes this requirement is neglected.) In particular, the reference of at least our simple concepts should supervene on something naturalistic, presumably causal, about the relation between our concepts and the referents of the concept (that can be a potential causal relation under (evolutionarily) relevant situations). ${ }^{15}$ If our conceptual resources are causally insulated from something, we would not be able to have simple concepts referring to those things.

Simple demonstrative concepts are simple concepts. If the phenomenal concepts are taken to be at least partly demonstrative concepts, and if we cannot have a demonstrative concept of something that we cannot have a simple demonstrative of, our demonstrative concepts and our phenomenal feelings must be able to stand in causal relation in order to establish the reference so that we can have the kind of demonstrative concepts the phenomenal concept strategy needs, as an advocate of the phenomenal concept strategy freely admits:

The denotation of a phenomenal type-demonstrative will be the property - presumably physical - that's causally responsible for the application of that concept in the introspective recognition or re-identification of an experience as 'that (kind) again' or 'another of those'. (Levin 2007: 89; her italics, my bolds)

Since some of the phenomenal feelings we instantiate are magnitudes, there is a causal barrier between such phenomenal feelings and non-magnitudes due to the proportionality requirement of causation. For any magnitude of pain, there is always a determinable, e.g., being very painful, that prevents the magnitude of pain from being a proportional cause of the instantiation of any non-magnitude (e.g., yelling). Therefore, based on what we have said about concept possession, there is a causal barrier between some of our phenomenal feelings and our concepts.

The causal barrier between the magnitudes and our conceptual resources would then imply that there can actually be no simple demonstrative concepts about those phenomenal feelings that are magnitudes. Hence, there can't be complex demonstratives about them as well. For that reason, no demonstrative concept can refer to such kind of phenomenal feeling from the physicalist perspective. The phenomenal concept strategy is not a viable physicalist response to the dualists. ${ }^{16}$

15 The usual thought is that once the reference of simple concepts is naturalized, we can explain reference of complex concepts with compositionality. For examples of attempts to naturalize simple mental representations, see Dretske (1981; 1988), Fodor (1987), Rupert (1999; 2001), Price (2001), Ryder (2004), etc.

16 Notice that this does not imply that phenomenal feelings have no causal power. Given what I have argued in this essay, my pain can still cause my enemy's pleasure (or my sadness) - for pleasure (or sadness) is a quantity that comes in degrees. And causal relations among various quantities appear to be exactly the kind of thing mathematical laws of nature purport to capture. I will leave causation among magnitudes of different quantities for another occasion. Neither does my argument imply that we cannot think about our phenomenal feelings at all; even a physicalist should not think that mental reference can only be established by a causal relation between a mental representation (e.g., a concept) and the referent. My argument only challenges the possibility of demonstratives about our phenomenal feelings. 
Note that my proportionality argument does not preclude demonstratives of, say, the determinable being very painful (instead of the maximally determinate way of being in pain). One might even include demonstratives of such determinables under the label 'phenomenal concepts', as Loar himself does (1990: section 10). But as I have argued extensively already in section 6, to respect the introspective datum that I feel only one pain, the less determinate mental property being very painful should not be considered identical to the painful feeling I instantiate - the subjective feeling of pain I instantiate is a maximally determinate mental property. Hence, a demonstrative about the less determinate mental property isn't a phenomenal concept that denotes what I subjectively feel when I am in pain. We need to keep in mind that the phenomenal concept strategy is supposed to use the idea of co-extensive concepts to explain away the apparent gap between the physical and the subjective feeling. And a point crucial to the strategy is the idea that we have two different kinds of concepts that refer to the same thing - our subjective feeling which is also a certain kind of brain state (by the physicalists' light). So, for the purpose of the strategy, the kind of demonstrative concepts involved ought to be about my subjective feeling. Bringing in less determinate demonstrative concepts about mental states that are not our subjective feelings, even if we choose to label these less determinate demonstrative concepts 'phenomenal concepts', would not help address the apparent explanatory gap between the physical and the subjective feelings, as far as the phenomenal concept strategy is concerned.

\section{Physicalist Resistance ${ }^{17}$}

According to my central claim, magnitude instantiations are too determinate to be proportional causes of demonstrative concepts for us to have demonstrative concepts about magnitudes. My explanation, as it is applied specifically to this case, can be captured by the following claim:

[*] When a magnitude $\mathrm{x}$ is instantiated and I generate the demonstrative concept $<$ this magnitude $>$, that same concept would have been generated just the same had a determinable of $x$ remained instantiated but the specific magnitude $\mathrm{x}$ failed to be instantiated (i.e., had a slightly different magnitude been instantiated instead).

A physicalist may try to reject this claim by saying that, had a slightly different magnitude been instantiated, a different concept would have been generated.

On what basis can our physicalist insist that a different concept would have been generated had a slightly different magnitude been instantiated? I suppose the reasoning is that the reference of a demonstrative concept is established by what causes it; if a slightly different magnitude is instantiated, it would be a different cause that leads to a different demonstrative concept. Notice that this argument goes through only if we assume that it is the magnitude that causes the concept; only by assuming that we can infer that the concept would be caused by something else (instead of by the same determinable) had a different magnitude been instantiated.

Now, with this observation, I'm not thereby suggesting that the physicalist pushback begs the question against me. I suppose that would be an uncharitable reading of what our physicalist

17 I would like to thank an anonymous reviewer for bringing some of the potential objections in this section to my attention. 
is getting at. Instead, I take her message to be that, since [*] cannot be true if magnitude instantiations can cause demonstratives, using [*] in my argument requires me to already have assumed that magnitude instantiations cannot cause demonstratives. It seems that $I$ am begging the question.

It isn't always straightforward to determine (if there is even a fact of the matter) who is begging the question against whom. If I argue against $\mathrm{q}$ based on $\mathrm{p}$, and if my argument is valid at all, of course my premise $\mathrm{p}$ would not be true if $\mathrm{q}$ is true. That alone shouldn't indicate that I'm begging the question. Here is why my argument isn 't begging the question.

Notice that [*] is strictly speaking not a premise I began with. Instead, as I said at the beginning of section 4 , '[w] hat I want to do is to figure out the reasonable thing to say with respect to the way magnitudes stand in causal relation with our concepts by giving a closer look at the way magnitudes stand in causal relation with other things.' By suspending my judgment hence not begging the question against anyone - about whether magnitude instantiations stand in causal relation with concept possession, I looked into other cases and obtained the general principle [Causal Profile] by induction. From that general principle, I can infer something like $\left[{ }^{*}\right]$, which goes on to tell us something about the nature of our phenomenal concepts.

Our physicalist can of course refuse to suspend the judgment with me and insist that the possession of mental demonstratives is a special case (i.e., rejecting [*]). On that bases, she flips my modus ponens for [*] into a modus tollens against [Causal Profile]. But one can always do that for any argument. Without a further independent reason for rejecting [*], this alone cannot be a legitimate objection to my argument. (Again, the physicalist pushback I'm considering is a piece of reasoning that relies on rejecting [*]; it is not a reason for rejecting [*].)

In addition to all that, it's also illuminating to consider what follows if one rejects [*] and embraces the view that our demonstratives can be caused by and thereby refer to individual magnitudes. Quantities come in very fine-grained degrees. Take a bath full of water as an example. Suppose I feel the water and say to myself 'this temperature is hot enough.' It takes time to entertain the thought. And it isn't crazy to think that the temperature of the water fluctuates slightly in that period even if the fluctuation is too small for me to sense. By our physicalist's own light, it seems that she has to say that I entertain a series of fluctuating thoughts with a series of distinct demonstrative concepts when I say to myself 'this temperature is hot enough' because of the fluctuating magnitudes. ${ }^{18}$ All things being equal, I find this way of characterizing the scenario implausible. I don't mean to present this as additional evidence for [*]. But this certainly adds significant weight to my earlier claim that our physicalist needs independent reason for rejecting $[*]$ instead of simply insisting that demonstratives about magnitudes are exceptions to my induction.

Alternatively, can our physicalist say that, by arguing that there are no demonstrative phenomenal concepts, I have concluded that there is no epistemic gap? Here's the thought. The epistemic gap is about a gap between our thoughts about the phenomenal and our thoughts about the

18 Of course, one can stipulate that I have a demonstrative concept that refers to the average temperature in that period. But such stipulations are made purely on pragmatic grounds. There is no epistemic reason for thinking that a demonstrative concept $<$ this temperature $>$ is about the average temperature instead of the average plus 0.001 degree Fahrenheit. 
physical - that we cannot infer the former from the latter. So, there is no epistemic gap between the phenomenal feelings and the physical if we can't have thoughts about the former. Since I have argued that the phenomenal feelings are maximally determinate and that we can't have demonstrative phenomenal concepts about such phenomenal feelings, there can be no epistemic gap related to the phenomenal feelings if we can't think about them. If the problem of an epistemic gap that haunts the physicalists could arise only for less determinate phenomenal properties, then the physicalists actually have no problem at all. In other words, I have thrown out the physicalist solution to the dualist problem along with the dualist problem itself. Far from being a problem for the physicalists, this is in fact a win for them.

Intuitively, there's an epistemic gap between our thoughts about what it is like to have a certain experience and our thoughts about brain states. We cannot obtain one from the other a priori. This is the epistemic intuition that fuels the Knowledge Argument. There are different ways to flesh out this epistemic intuition in order to explain it. One might appeal to the different kinds of object — physical vs. phenomenal properties - that our concepts can be about. That is what the dualists think we should do. Alternatively one might appeal to the different kinds of concept that we can have about the same objects. The phenomenal concept strategy takes the latter approach and, on top of that, tries to explain the special features that the what-it-is-like of our experience appears to have in terms of the special features of demonstrative concepts.

Here are two things that I didn't argue against. First of all, I didn't argue against the intuition of an epistemic gap. Arguing that one cannot describe and explain the intuition the way many physicalists thought they can is not to argue that the intuition isn't there or that it need not be explained. Secondly, I didn't argue against fleshing out the intuition in terms of different kinds of concept. My argument leaves it open for us to choose whether we decide to flesh out the epistemic intuition in terms of the differences in object-types or in terms of the differences in concept-types. I only argue that we cannot think about magnitudes with demonstratives - a specific kind of concept whose references must be fixed causally by physicalist light. Hence, I didn't argue that we cannot think about phenomenal feelings, just not with demonstratives. Unless the advocates of the phenomenal concept strategy refuses to acknowledge that there are other ways to flesh out and explain the intuitive epistemic gap, my argument against their way shouldn't be understood as an argument that undercuts the dualist problem because the epistemic gap between our thoughts about the phenomenal and our thoughts about the physical is still there to be explained.

Finally, given that we appear to refer to our phenomenal feelings with demonstratives quite often, one might consider what I have just said to be an objection to physicalism about mental representation. One can take it that way (which will then be a separate argument against physicalism), but one does not have to. And I don't. I remain neutral on that issue, at least in this essay. There are a lot of things a physicalist can say even if she grants everything I have just said about our mental demonstratives. She might think that, like a lot of our thoughts in everyday life, apparent demonstratives about phenomenal feelings should not be taken literally; instead, they are just a pretense of demonstrative thoughts for pragmatic purposes. The only point I want to make is this: It seems right that at least some phenomenal qualities are quantities; if so, a proper metaphysics of quantity implies that there are no physicalism-friendly demonstrative concepts 
about phenomenal feelings for the phenomenal concept strategy to work. This is not a problem that can be fixed simply by beefing up the phenomenal concepts. ${ }^{19}$

\section{References}

Alter, T. \& Walter, S. (eds.) 2007. Phenomenal Concepts and Phenomenal Knowledge. OUP. Armstrong D. M. 1988. 'Are Quantities Relations? A Reply to Bigelow and Pargetter,' Philosophical Studies 54(3): 305-316.

Ball, D. 2009. 'There are No Phenomenal Concepts.' Mind 118: 935-962.

Bigelow J. \& Pargetter R. 1988. 'Quantities,' Philosophical Studies 54(3): 287-304.

Block, N. 2002. 'The Harder Problem of Consciousness'. Journal of Philosophy 99: 1-35.

Byrne, A. 2003. 'Color and Similarity'. Philosophy and Phenomenological Research 66(3): 641665.

Byrne, A. \& Hilbert, D. 2003. 'Color Realism and Color Science'. Behavioral and Brain Sciences 26(1): 3-21.

Caruthers, P. 2004. 'Phenomenal Concepts and Higher-Order Experiences.' Philosophy and Phenomenological Review LXVIII(2): 316-336.

Dasgupta, S. 2013. 'Absolutism vs Comparativism About Quantity.' Oxford Studies in Metaphysics 8: 105-148.

Dretske, F. 1981. Knowledge and the Flow of Information. MIT.

- 1988. Explaining Behavior: Reason in a World of Causes. MIT.

Eddon, M. 2013. "Quantitative Properties.” Philosophy Compass 8: 633-645.

Ellis, B. 1966. Basic Concepts of Measurement. Cambridge University Press.

Fodor, J. 1987. Psychosemantics. MIT.

Forge, J. (ed.) 1987. Measurement, Realism and Objectivity. Springer.

—. 1987. 'Ellis' Theory of Quantities.' in Forge (ed.) (1987): 291-309.

Jackson, F. 1982. 'Epiphenomenal Qualia,' in Philosophical Quarterly 32: 127-136.

Levin, J. 2007. 'What is a Phenomenal Concept?' in Alter \& Walter (eds.) (2007): 87-110.

Levine, J. 2007. 'Phenomenal Concepts and the Materialist Constraint'. in Alter \& Walter (eds.) (2007): 145-166.

—. 2010. 'Demonstrative Thought.' Mind \& Thought 25(2): 169-95.

Loar, B. 1990. 'Phenomenal States.' Philosophical Perspectives 4, Action Theory and Philosophy of Mind: 81-108.

Mundy, B. 1987. 'The Metaphysics of Quantity.' Philosophical Studies 51(1): 29-54.

Price, C. 2001. Functions in Mind: A Theory of Intentional Content. OUP.

Rupert, R. 1999. 'The Best Test Theory of Extension: First Principle(s)'. Mind \& Language 14: $321-355$.

_. 2001. 'Coining Terms in the Language of Thought: Innateness, Emergence, and the Lot of Cummins's Argument against the Causal Theory of Mental Content'. The Journal of Philosophy 98(10): 499-530.

19 See also Ball (2009) for another recent attempt to argue that there is no such thing as phenomenal concepts. 
Ryder, D. 2004. 'SINBAD Neurosemantics: A Theory of Mental Representation'. Mind \& Language 19: 211-240.

Shapiro, L. \& Sober, E. 2012. 'Against Proportionality.' Analysis 72(1): 89-93.

Swoyer, C. 1987. 'The Metaphysics of Measurement.' in Forge (ed.) (1987): 235-290.

Tye, M. 2000. Consciousness, Color, and Content. OUP. -. 2003. 'A Theory of Phenomenal Concepts.' Royal Institute of Philosophy Supplement 53: 91105.

-. 2009. Consciousness Revisited. OUP.

Schroer, R. 2010. 'Where's the Beef? Phenomenal Concepts as Both Demonstrative and Substantial'. Australasian Journal of Philosophy 88(3): 502-522.

Siegel, S. 2010. The Contents of Visual Experience. OUP.

Wilson, J. 2009. 'Determination, Realization and Mental Causation.' Philosophical Studies 145(1): 149-169.

Yablo, S. 1992. 'Mental Causation.' Philosophical Review 101(2):245-280.

—. 1997. 'Wide Causation.' Philosophical Perspectives 11(11): 251-281. 